\title{
Percutaneous Full-endoscopic Translamina Fenestration Approach for Treatment of A Lumbar Nerve Root Cyst and Literature Review
}

\author{
Weijun Kong ${ }^{1,2}$, Zhijun Xin ${ }^{1}$, Qian Du ${ }^{1}$, Taiyong Chen ${ }^{1,2}$ and Wenbo Liao ${ }^{1 *}$ \\ ${ }^{1}$ Department of Spine Surgery, The Affiliated Hospital of Zunyi Medical University, Guizhou China \\ ${ }^{2}$ Department of Orthopedics Surgery, West China Hospital, Sichuan University, Sichuan China
}

*Corresponding author: Wenbo Liao, Director of the Orthopedic Center, The Affiliated Hospital of Zunyi Medical University, No.1

Xinpu Road, Zunyi, 563000, Guizhou China

\section{ARTICLE INFO}

Received: 㓞 July 08, 2019

Published: 彗 July 16, 2019

Citation: Weijun Kong, Zhijun Xin, Qian Du, Taiyong Chen, Wenbo Liao. Percutaneous Full-endoscopic Translamina Fenestration Approach for Treatment of A Lumbar Nerve Root Cyst and Literature Review. Biomed J Sci \& Tech Res 19(4)-2019. BJSTR. MS.ID.003338.

\begin{abstract}
Here we report on a percutaneous full-endoscopic translamina fenestration approach combined Radio frequency (RF) for the treatment of lumbar nerve root cyst with a successful clinical outcome. A 28-year- old man presented with left knee, dorsumthigh pain , numbness. left knee joint extension is anemic, and left knee tendon reflex is weakened due to a sheath cyst at the L3 nerve root. The procedure of percutaneous fullendoscopic translamina fenestration approach was used to remove the cyst, achieving complete decompression of the nerve root. The symptoms were relieved, and the patient was discharged at the postoperative third day. The Magnetic resonance imaging(MRI) obtained postoperatively, showing no residual cystic mass and the decompressed L3 nerve root. A percutaneous endoscopic vertebral plate fenestration approach using RF appears to be a suitable alternative treatment for selected patients with lumbar nerve root cysts.
\end{abstract}

\section{Introduction}

Physiological basis of epidural nerve root cyst formation is certain developmental vulnerabilities of nerve root sleeve [1]. Cystic growing biological mechanisms is from human walked upright with high hydrostatic pressure and the effectiveness of ball valve $[1,2]$. As the cyst progresses, it may exert a significantly effect on the surrounding nerve root or dural sac. The authors analyzed a rare case of a nerve root cyst that located in L2-3 intervertebral foramen, and here we report the usefulness of a percutaneous fullendoscopic translamina fenestration approach combined RF for the cyst.

\section{Case Report}

A 28-year-old man presented with chief complaints of left dorsum-thigh numb-ness, feeble and radicular pain. Physical examination demonstrated a positive left femoral nerve pull test sign. There was objective weakness of the rectus femoris grade as 2-3. The knee reflex also was decreased on the left side. VAS:6.0.
The patient had no fasciculation, muscle atrophy, or upper motor neuron signs. Magnetic resonance imageing (MRI) showed an intraspinal extradural space-occupying lesion in L2-3 intervertebral foramen on the left side. The irregular oval-shaped lesion was hypointense on a CT image and hyperintense on a T2-weighted sequence, suggesting a cystic lesion (Figure 1). A percutaneous fullendoscopic translamina fenestration procedure was performed under general anesthesia with the patient in the prone position on a radiolucent table under the guidance of C-arm fluoroscopy.

First of all, the mid-line was marked with a pen on the patient's back surface. the medial pedicular line at the L2 vertebral level and the inferior edge of the L2 vertebral body were marked under image intensification, and body surface projection point of L2-3 left intervertebral foramen was decided, positioning needle was introduced and followed by expansion of the guide bar to the L2 vertebral plate, with a ring saw to establish bone windowl of work casing by the vertebral plate drilling (Figure 2), take away 
the window bone. The working cannula was introduced via the vertebral plate fenestration (Figure 3). Epidural fat, epidural fibrotic tissue were removed and cleared off using endoscopic forceps, a radiofrequency electrode. After clearing for endoscopic visualization, the surgeons could find the cyst and com-pressed nerve root. Which was a well-circumscribed reddish mass located at the axillary portion of the nerve root and occupied the intervertebral foramen. since the lesion was attached to the nerve root sleeve. using a radiofrequency electrode, adhesion around the cyst were removed initial, some of the lesion was removed using RF and endoscopic forceps without neural damage, and the remaining portion of the cyst at the axillary of nerve root sleeve was coagulated and removed using a radiofrequency electrode.
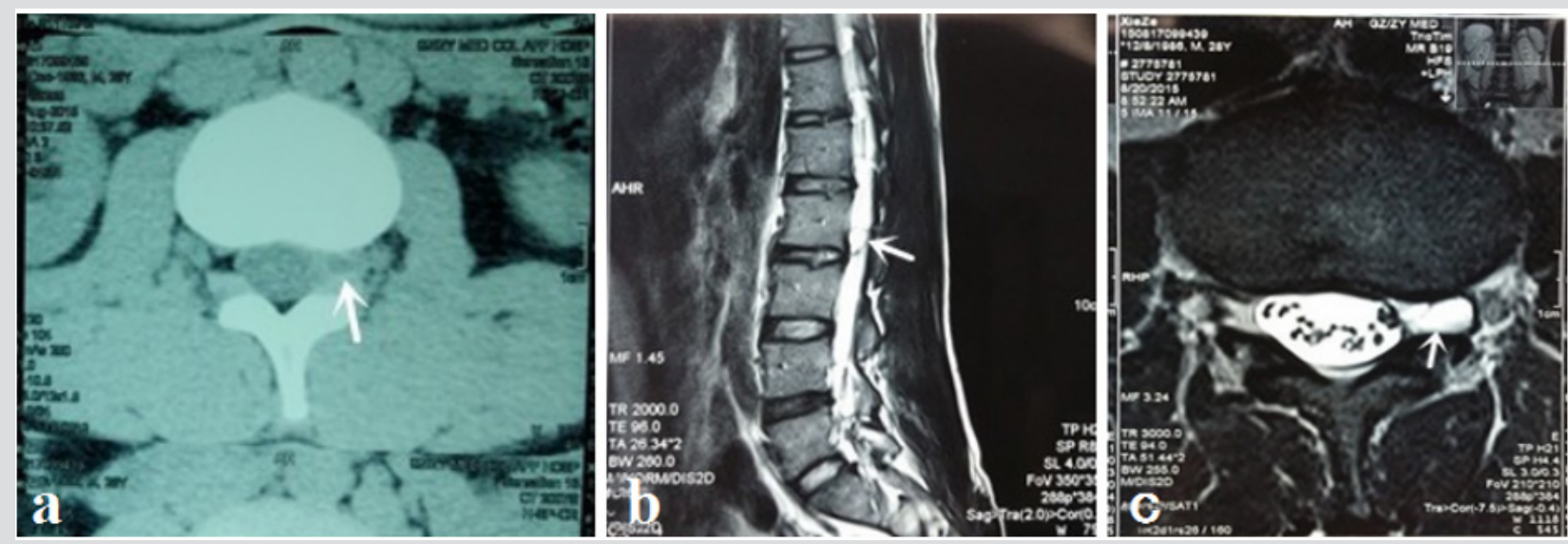

Figure 1: (a)Preoperative CT image showed a cystic lesion with low signal intensity(white arrows) at the L2-3 intervertebral foramen. (b) preoperative sagittal T2-weighted MRI images showed a cystic lesion with high signal intensity(white arrows). (c) preoperative axial T2-weighted MRI images showed a cystic lesion with high signal intensity (white arrow) compressing the L3 nerve root.

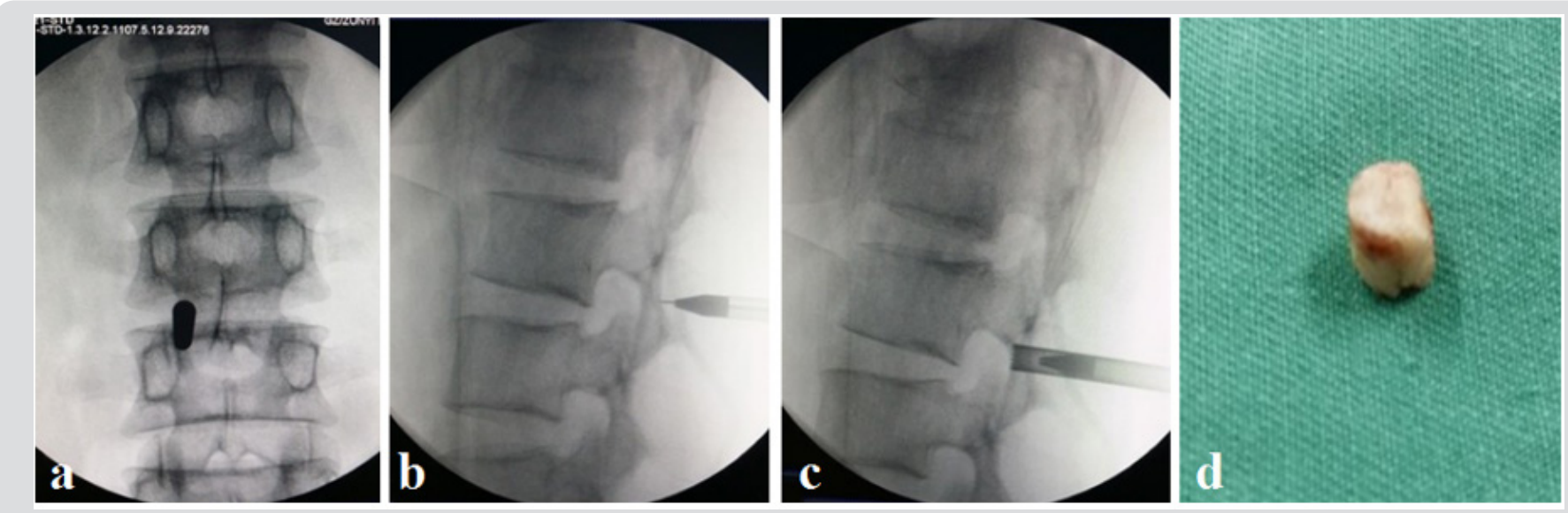

Figure 2: $(\mathrm{a}-\mathrm{c})$ Intraoperative intensifier images showing the placement of the cannula and trephine at the vertebral plate in anteroposterior and lateral views; (d) taked the window bone.

And the electrode coagulation blocks off the leak of cerebrospinal fluid to the cyst. After the procedure, we could see the decompressed nerve root (Figure 3).one day after the operation, the patient was ambulatory without feeble and pain, demonstrated the L3 nerve root was successfully decompressed, and the result was confirmed by postoperative MRI images (Figure 4a) Pathological studies showed a collagenous fibrous wall of a nerve root cyst without no synovial lining cell of inflammatory cell infiltration (Figure 4b). Two years follow-up of postoperative, the patient did not complain of back pain, leg pain, numbness or other discomfort in the lower limbs during the out-patient followup examination, VAS:0. Lumbar MRI showed no recurrence of cysts and no abnormality of local spinal canal (Figure 5). 


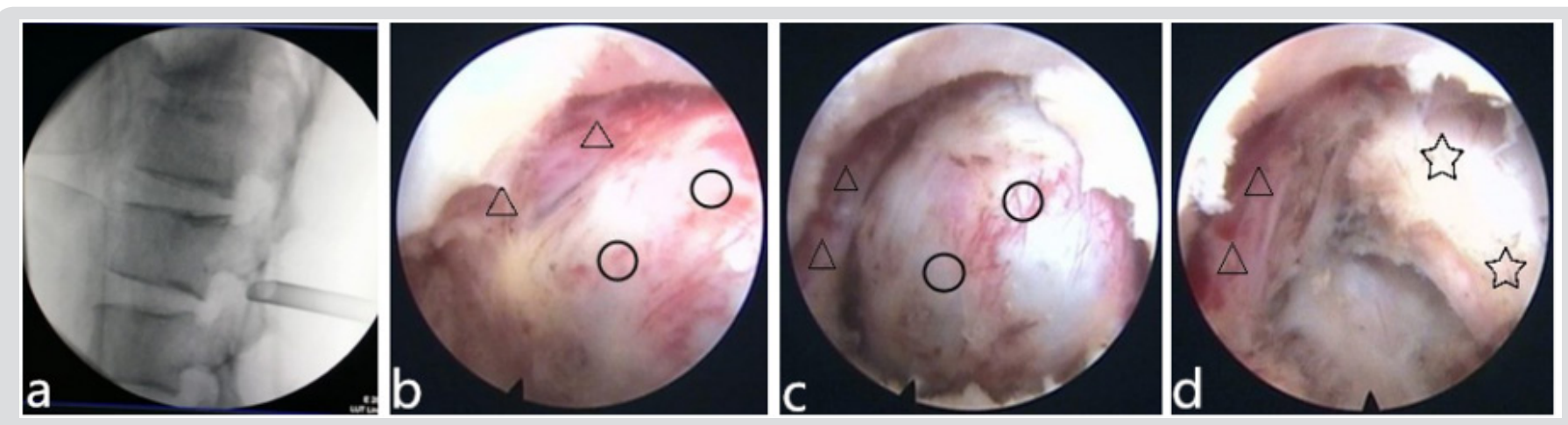

Figure 3: (a)Lateral X-ray to determine the working casing depth; (b-d)Full-endoscopic view showed the cystic mass( $\circ)$, the compressed L3 nerve $\operatorname{root}(\Delta)$; and intraoperative removed cyst fibous wall, exposured dural sac(光).

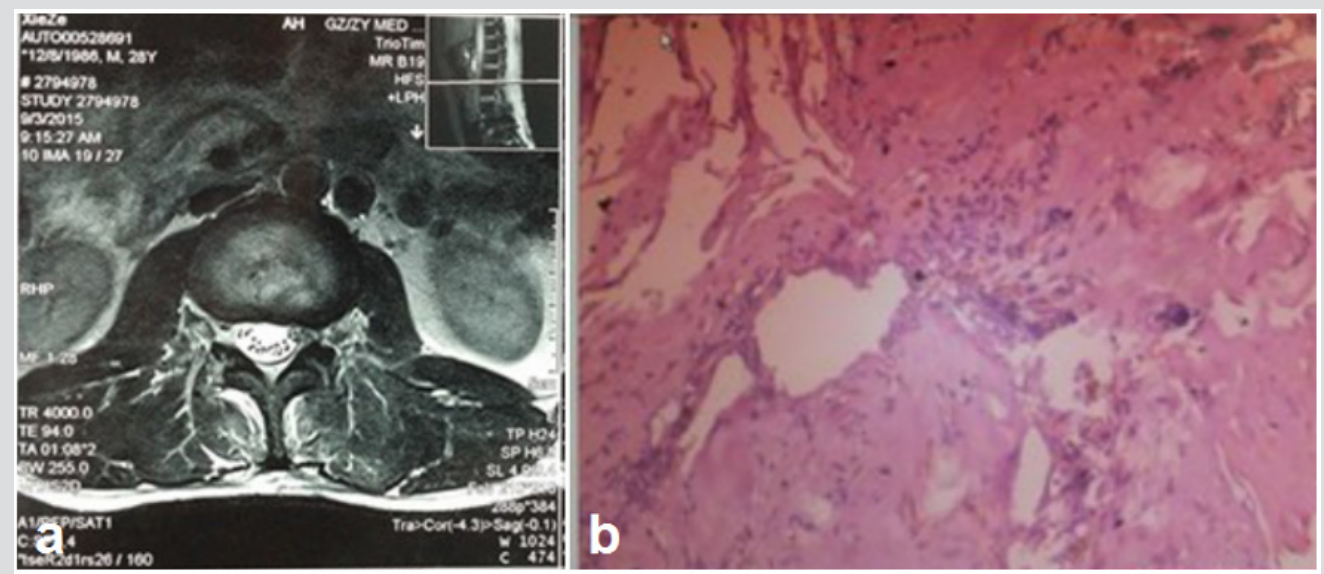

Figure 4: (a)Postoperative axial T2-weighted MRI image showed no residural cystic mass and the decompress; (b)Postoperative pathological studies showed a collagenous fibrous wall of nerve root cyst.
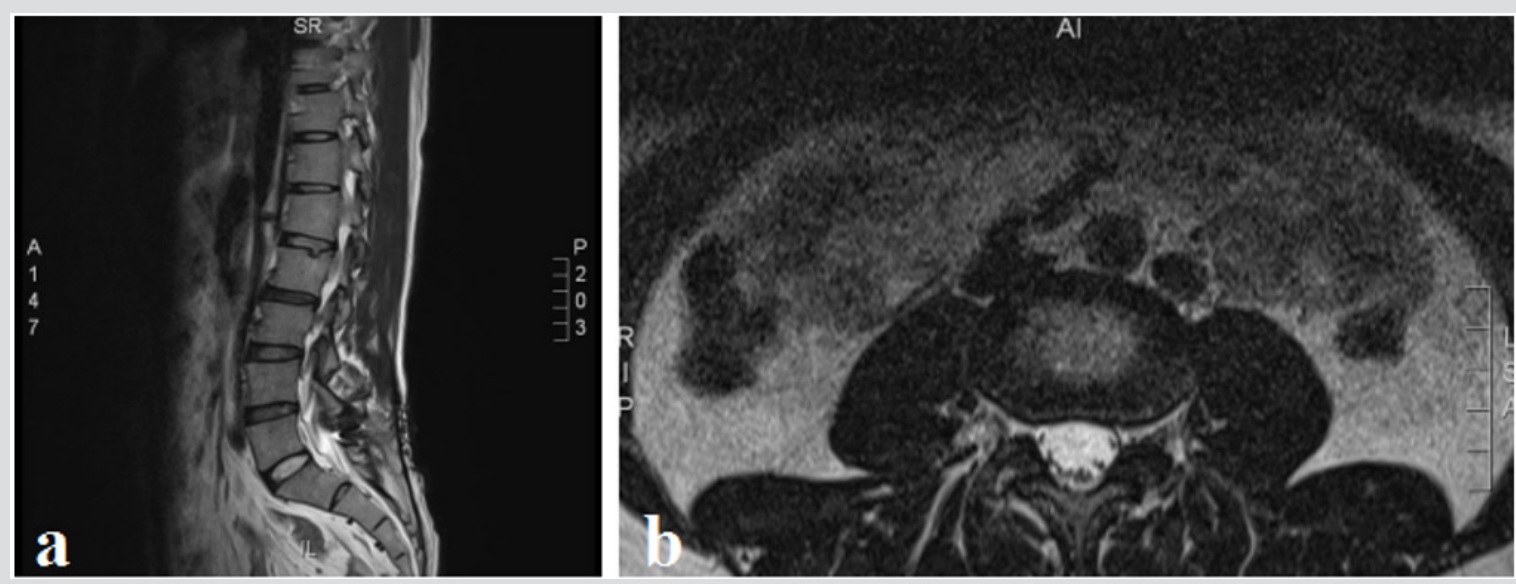

Figure 5: Lumbar MRI showed no recurrence of cysts and no abnormality of local spinal canal at postoperative 2-years followup.

\section{Discussion}

There are many kinds of cystic lesions affecting the spinal canal, including arachnoid cysts, sacral extradural spinal meningeal cysts, synovial cysts, ganglion cysts, cysts of the ligament flavum, perineural cysts, and nerve root cysts [4-7]. Sacral spinal meningeal cysts are common in the clinical work, but a nerve root cyst is uncommon and has rarely been reported in the literature $[6,7]$. Some scholars believe that the initiating factors of the cyst formation is the hydrostatic pressure of cerebrospinal fluid $[8,9]$. Due to nerve root sleeve developmental abnormalities, and local connective tissue disorder structure, there are small cracks or fistula. formed a one-way valve structure. cough actions cause the local hydrostatic pressure increased in Dural sac; cerebrospinal fluid infiltrated into cyst $[10,11]$. At the resting state, the Dural sac hydrostatic pressure drop, but the cyst fluid backflow stimulates mucous membrane of the cyst seal leaks, cyst fluid cannot back to 
normal circulation by leak, lead to cyst increase gradually [12]. In the process of cyst development and growth, adjacent nerve root was irritant and compression. The corresponding symptoms were dis-played, more shows the dominated regional pain of compressed nerve root, and numbness, hypesthesia, even with muscle strength decreased.

It was a consensus that asymptomatic cyst does not require surgical intervention [10-12], but as symptoms of cyst patients, the purpose of surgical intervention is resecting cysts and relieve nerve root stimulation and remove compression. Various methods for removal of spinal cysts have been used for treatment of the condition. Surgical methods including surgical excision and percutaneous CT-guided aspiration and steroid injection have been reported, which have been favorable clinical outcomes of these treatments [13]. As open surgery leads severe trauma. Min et al. attempted to move a synovial cyst of the facet joint in the lumbar spine by a percutaneous endoscopic transforaminal approach and achieved excellent pain relief [14].

To our knowledge, however, no study has previously reported on an percutaneous full-endoscopic translamina fenestration approach with RF for lumbar nerve root cyst. Since the cyst manifested symptoms and signs in accordance with the irritant of L3 nerve root. In order to avoiding open surgical trauma and complications. We considered a minimally invasive percutaneous full-endoscopic procedures for the treatment of the nerve root cyst. Through careful analysis and accurate positioning, percutaneous by lamina building window as working channel straight for the cyst. Full-endoscopic Radio-frequency condensed cyst step by step and closed fistula with electric coagulation, achieved good nerve root decompression. the procedure was success-fully accomplished, L3 nerve root was successfully decompressed and the result was confirmed by postoperative MRI imaging.

\section{Conclusion}

In this report, we describe a minimally invasive procedure that a percutaneous full endoscopic lamina fenestration approach with Radiofrequency to remove a nerve root cyst in the L2-3 intervertebral foramina with a successful outcome. Therefore,

\section{ISSN: 2574-1241}

DOI: 10.26717/BJSTR.2019.19.003338

Wenbo Liao. Biomed J Sci \& Tech Res

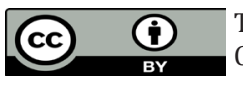

This work is licensed under Creative Commons Attribution 4.0 License

Submission Link: https://biomedres.us/submit-manuscript.php we suggest that percutaneous endoscopic lamina fenestration approach is an effective and safe means of treating of nerve root cyst in selected cases.

\section{References}

1. Christoph Scholz, Ulrich Hubbe, Evangelos Kogias, Klingler JH (2015) Incomplete resection of lumbar synovial cysts - Evaluating the risk of recurrence. Clin Neurol Neuro surg 136: 29-32.

2. Takashi S, Saburo Y, Shigeo M (2015) Pathological characteristics of cyst formation following gamma knife surgery for arteriovenous malformation. Acta Neurochirurgica 157(2): 293-298.

3. Langdown AJ, Grundy JR, Birch NC (2005) The clinical relevance of Tarlov cysts. J Spine Discord Tech 18(1): 29-33.

4. Doyle AJ and Merrilees M (2004) Synovial cysts of the lumbar facet joints in a symptomatic population: prevalence on magnetic resonance imaging. Spine 29(8): 874-878.

5. Wildi L M, Kurrer MO, Benini A, Weishaupt D, Michel BA, et al. (2014) Pseudocystic degeneration of the lumbar ligamentum flavum: a little know entity. J Spine Disord Tech 17(5): 395-400.

6. Park H J, Kim S, Lee S W, Byung Chul Son (2008) Two cases of symptomatic perineural cysts (Tarlov cysts) in one family. A case reports. J Korean Neurosurg Soc 44(3): 174-177.

7. Ju C, Shin H, Kim SW, Hyeun Sung Kim, et al. (2009) Sacral perineural cyst accompanying disc herniation. J Korean Neurosurg Soc 45(3): 185187.

8. Fernandes C, Pinho R, Veloso R, Pinto Pais T, Carvalho J, et al. (2012) Tarlov cysts: an unusual case of perianal pain. Tech Coloproctol 16(4): 319-320.

9. Pena E, Lanero M (2011) Painful legs and moving toes syndrome associated with a sacral Tarlov cyst. Parkinsonism Related Disord 17(8): 645-646.

10. Cantore G, Bistazzoni S, Esposito V, Tola S, Lenzi J, et al. (2013) Sacral Tarlov cyst: surgical treatment by clipping. World Neurosurg 79(2): 381389.

11. Voyadzis JM, Bhargava P, Henderson FC (2001) Tarlov cysts: a study of 10 cases with review of the literature. J Neurosurg 95 ( 1 Suppl 1): 25-32.

12. Landers J, Seex K (2002) Sacral perineural cysts: imaging and treatment options. Br J Neurosurg 16(2): 182-185.

13. Koga H, Yone K, Yamamoto T, Komiya S (2003) Percutaneous CT-guided puncture and steroid injection for the treatment of lumbar discal cyst: a case report. Spine 28(11): E212-E216.

14. Min JH, Chung BJ, Lee SH (2006) Endoscopically managed synovial cyst of the lumbar spine. Korean J Spine 3: 242-245.

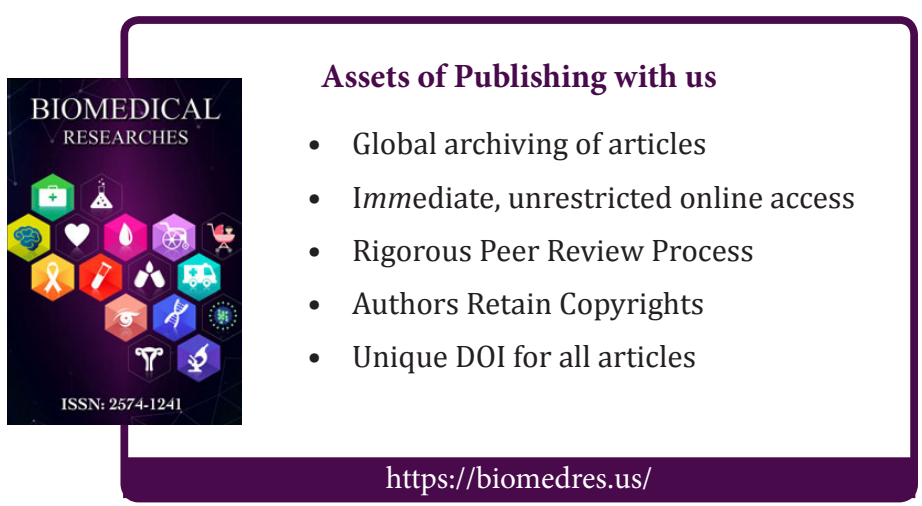

